\title{
To Examine the Relationship and Strength of Alcohol- Related Intimate Partner Violence in sub-Saharan Africa
}

\author{
Ekpenyong $\mathrm{MS}^{1 *}$, Awa Uchechi ${ }^{2}$ and Nzute $\mathrm{A}^{3}$ \\ ${ }^{1}$ Department of Public Health, University of Wolverhampton, United Kingdom \\ ${ }^{2}$ Department of Public Health Faculty of Education, Birmingham-city University, United Kingdom \\ ${ }^{3}$ Department of Public Health, University of Wolverhampton, United Kingdom
}

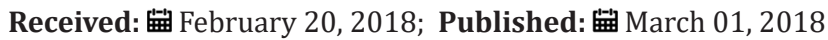

*Corresponding author: Ekpenyong MS, Department of Public Health, Faculty of Education, Health and wellbeing, University of Wolverhampton, United Kingdom

\begin{abstract}
Alcohol-related intimate partner violence (IPV) is a serious public health issue which has attracted a lot of research and debates. While some studies have reported the relationship between alcohol and IPV to be linear, others have reported threshold effects. While some studies have found the link to be strong, others have reported weak or no association. Using Logistic regression and meta-analysis, the relationship, strength of relationship and possible moderators of the alcohol-IPV link are investigated in ten sub-Saharan African countries. The results indicates that while alcohol consumption is associated with IPV in three of the countries, alcohol abuse was associated with IPV in the other seven countries lending support for both the linear and threshold effects in sub-Saharan Africa. The meta-analysis showed a strong association between alcohol and physical IPV while a weaker association was observed for the alcoholsexual IPV link. Moderator analysis showed that the strength of the alcohol-IPV link in sub-Saharan Africa varies with wealth index, marital length, and marital status, and jealousy, place of residence and justification of the use of violence. The nature of moderation was different between countries. The results of this study can be applied to plan country specific and multi-faceted intervention programs.
\end{abstract}

Keywords: Alcohol; Intimate Partners; Violence; Sub-Saharan Africa

\section{Introduction}

Intimate partner violence (IPV) is defined as any physical, psychological or sexual harm that is caused by the actions of a present or previous intimate partner [1]. It a major public health issue and violates women's human rights [1]. Cross sectional studies have shown that 10-69 per cent of women of reproductive age experience physical violence at least once in their lifetime while 6-59\% report an attempt or actual sexual violence by their intimate partners [2]. Intimate partner violence takes place in all backgrounds and among all socioeconomic, religious and cultural groups with women bearing the global burden [1]. Prevalent rates are different across countries with rates between $11-52 \%$ in developing countries [3]. IPV has been reported to lead to physical injuries, loss of pregnancy and complications during pregnancy [2]. It can also result in emotional problems such as depression and suicide [2] and victims have been reported to resort to use of drugs and alcohol as a means of coping with the abuse [4].
Several risk factors such as young age, low education, occupation, experiencing parental violence, drug and alcohol use, controlling behaviour by the husband [5], justification of wife beating [6] and so on has been reported to increase the odds of IPV; of these, alcohol consumption has been consistently implicated $[2,7]$ with the prevalence of alcohol-related IPV differing in diverse countries [2]. Although an association between alcohol and IPV has been established in previous studies, there are arguments on the role of alcohol in IPV, the effect of alcohol and the strength of the association between alcohol and IPV. Although alcohol-related IPV is a widely researched topic, only some research has been done in sub-Saharan Africa [8,9] and none has investigated the magnitude of the association across countries in Sub-Saharan Africa. The aim of this study was to determine the type of association between alcohol and IPV in sub-Saharan Africa and also examine the strength of the relationship between alcohol and IPV in Sub-Saharan Africa. 


\section{Methodology}

For the quantitative study, secondary analysis and meta-analysis were used to analyze cross-sectional data from the demographic and health surveys of ten countries in sub-Saharan Africa (Burkina Faso, Ghana, Kenya, Liberia, Malawi, Nigeria, Sao Tome and Principe, Tanzania, Zambia and Zimbabwe). Since the aim of this research was to determine the relationship between alcohol and IPV in sub-Saharan Africa, a quantitative research design was adopted because it is an appropriate method for showing associations and quantifying relationships between variables [10]. In order to examine the relationship and moderators of the alcohol-IPV link in sub-Saharan Africa, a secondary analysis of previously conducted primary studies of ten countries in sub-Saharan Africa was carried out. This is the method of choice for this research as it takes a cross national perspective which requires that data from several countries in sub-Saharan Africa be analysed. Data sets for this study were also easier to access and raise little or no ethical issues as respondents are already made anonymous.

\section{Data Collection Methods}

This study did not collect primary data but accessed data of the demographic and health survey of ten sub-Saharan Africa countries (Burkina Faso, Ghana, Kenya, Liberia, Malawi, Nigeria, Sao Tome and Principe, Tanzania, Zambia and Zimbabwe). Large sample sizes were used with high response rate thereby ensuring that statistically significant relationships are detected. Access to the data sets was gained by requesting permission from Demographic and Health Survey (DHS). Approval from DHS was granted by email. Data was identified using the domestic violence questionnaire. The identified data sets were downloaded to the researcher's personal computer using SPSS (version 19) software. Variables in the study were identified using the DHS recode manual.

\section{Sample/Sampling Strategy}

This study used quota sampling to identify DHS surveys conducted between 2006 and 2011 made available by 2012 in sub-Saharan Africa. For this research, only countries from sub-Saharan Africa were included because the main independent variable (alcohol consumption) was not measured in North African countries as consumption is prohibited. The data for each country was the most recent. This was to ensure that results reflected the current strength of the alcohol-IPV link and that recommendations are made based on current evidence. All datasets included asked questions on domestic violence and covered the topic of alcohol consumption and frequency at which husband/partner gets drunk because of the fact that the focus of this study is on alcohol-related IPV.

\section{Data Analysis}

Data analysis was carried out using SPSS (version 19.0) and Revman Meta-analysis software.

Univariate Analysis: Frequencies were used to determine the prevalence of the different forms of IPV, alcohol consumption and alcohol related IPV in the ten countries included in this study.

Logistic Regression: In order to determine the type of relationship between alcohol and intimate partner violence in sub-Saharan Africa, a logistic regression of the four category alcohol measure was carried out by comparing non-drinkers to drinkers (drinkers who never got drunk, who got drunk sometimes and those who got drunk often). Results are reported as B (Standard Error), odd ratios (OR) and 95\%CI for OR. A significant Wald test p-value indicates a significant difference between the categories and non-drinkers.

Meta-Analysis: In order to investigate the strength of the alcohol-IPV link in sub-Saharan Africa, a meta-analysis was carried out using the Revman software. This was done by comparing non-abusers (non-drinkers and drinkers who never got drunk) and abusers of alcohol (husbands who got drunk sometimes and often). Based on the assumption that effects may vary across samples and studies [11], random effects model was used. The random effects model was used in this study because of the heterogeneous nature of the studies and because this model generates results that are generalisable to the sub-Saharan Africa population. The heterogeneity of the result was investigated using the Cochran's $Q$ test [12]. A significant I2 shows heterogeneity among included studies with higher values indicating increased differences within study [12]. Due to the high heterogeneity between countries included in the meta-analysis, further moderation analysis was carried out.

Hierarchical Logistic Regression: Using the method described by Field [13], hierarchical logistic regression was used to study the moderator effects of the independent variables on the alcohol-IPV link. In order to account for the complex sampling used in the DHS survey, the probability of being administered the domestic violence questionnaire and to adjust for the probability of non-response [14]; all analyses were conducted using the domestic violence sample weight.

\section{Ethical Issues}

One of the ethical issues with conducting a secondary data analysis is the permission to access datasets. Approval was sought by the researcher and access was granted. The DHS also operate a no data sharing policy. To ensure data protection, memory sticks and computers were password protected to guard against unauthorised access. It is also important to confirm that the data obtained from the primary study was ethically obtained. The primary DHS study was done with the informed consent of the subjects and confidentiality obtained with the National ethics committees of the different countries approving the surveys. Furthermore, the datasets have been made anonymous by removing all identifier information.

\section{Results}

The study population consisted of women aged 15-49 years old and men aged 15-59 years old in ten countries in sub-Saharan Africa. Six hundred and eighty-two couples were interviewed in Sao 
Tome and Principe, 873 in Tanzania and 883 in Ghana. The largest numbers of 5566 couples were included in Nigeria while 3488 and 3051 couples participated in Burkina Faso and Malawi respectively (Figure 1). Of these numbers, the highest percentage of women were in the 25-29 years' age group with this group accounting for $22.1 \%$ of the women in Burkina Faso, 27\% in Kenya, 25.8\% in Ma- lawi and $27.7 \%$ in Tanzania. For the men, 22.6\%, 20.8\%, 21.3\% and $21.4 \%$ were in the 30-34 years' age group in Kenya, Malawi, Zimbabwe and Zambia respectively. In Ghana (19.8\%), Liberia (21.5\%) and Nigeria (19.8\%), the highest proportion of the male participants were in the 35-39 years' age group (Figures 2a \& 2b).

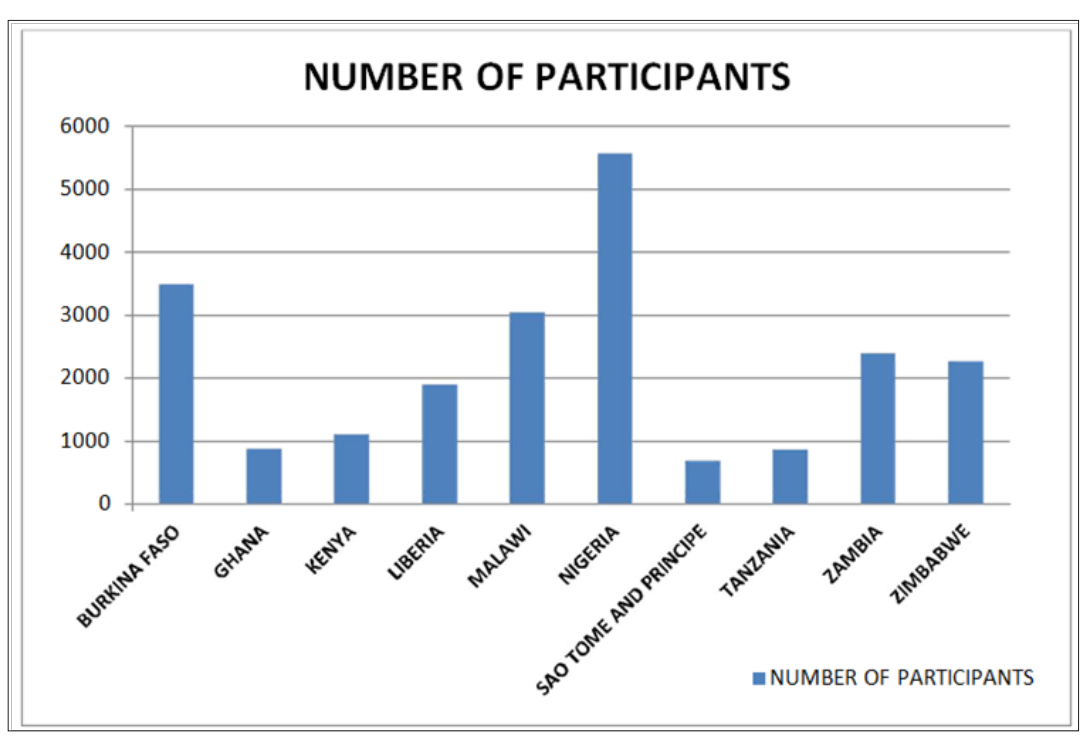

Figure 1: Number of Participants in the Study.

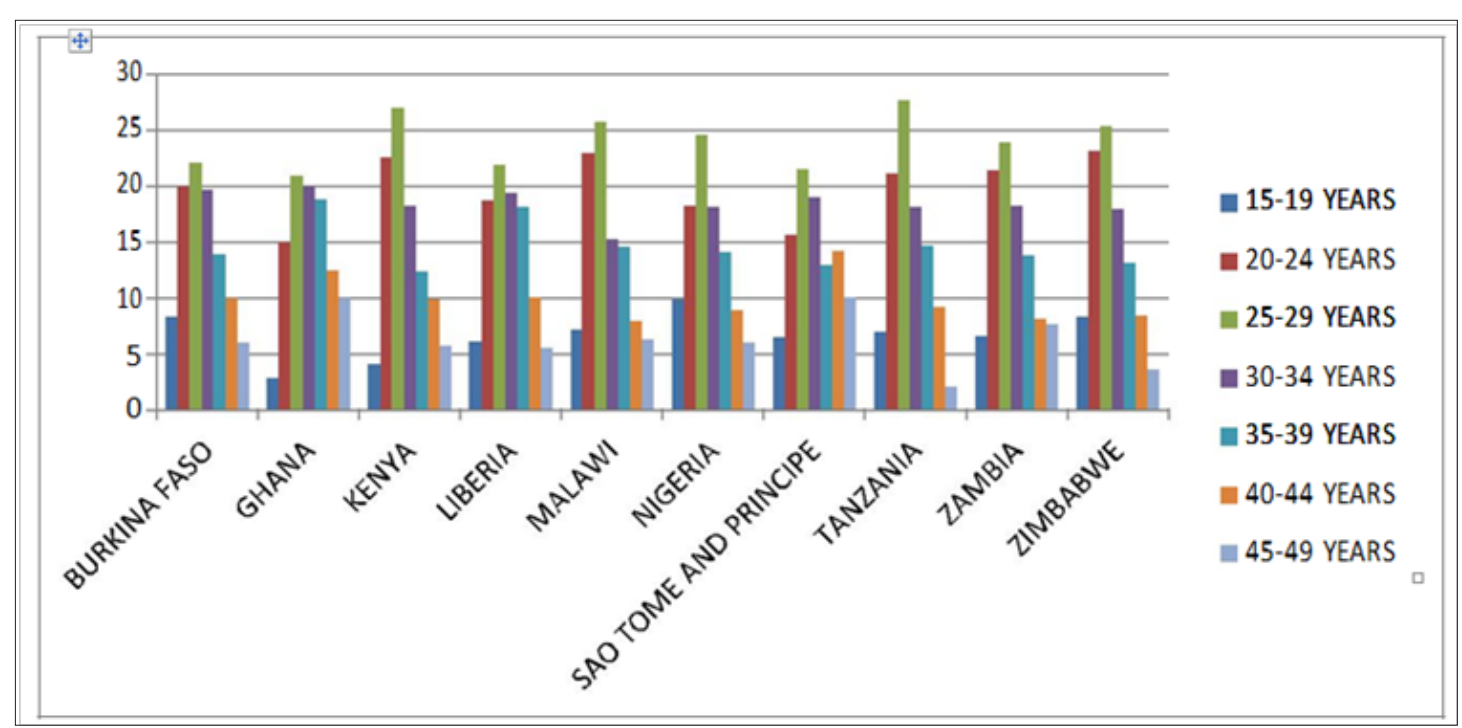

Figure 2a: Age group of the Women.

In Burkina Faso, $82.3 \%$ of the women had no education compared to $77.0 \%$ of the men. Zimbabwe and Sao Tome and Principe were found to have the highest number of couple who have had any form of education with $94.2 \%$ of the women and $99.4 \%$ of the men having a primary, secondary or higher education in Sao Tome and Principe. In Zimbabwe, only $2.7 \%$ of the women and $1.2 \%$ of the men have had no education at all. $5.2 \%$ of the women in Burkina Faso and $6.6 \%$ of the women in Tanzania reported having a second- ary education compared to $41.5 \%$ and $62.1 \%$ in Ghana and Zimbabwe respectively (Figures 3a \& 3b).

\section{Employment Status}

Highest proportions of women who are unemployed were in Zimbabwe, Kenya, Zambia and Malawi with 62.3\%, 61.6\%, 48.3\% and $42.3 \%$ of the women reporting that they have no paid employment respectively. The highest number of women who were em- 
ployed was reported in Ghana (88.5\%), Tanzania (86\%), Burkina Faso $(78.6 \%)$ and Nigeria $(64.4 \%)$. For the men, the highest rate of unemployment was in Zimbabwe (24.2\%), Malawi (6.9\%) and Liberia (6.3\%) (Figure 4). When wealth index was considered, Figures 5 show that $24.5 \%$ and $22.3 \%$ of Nigerians in the study popu- lation were in the poorest and poorer wealth indexes respectively. Kenya, Sao Tome and Ghana have the highest percentages of $30.2 \%$, $25.4 \%$ and $23.4 \%$ of the couples in the richest wealth index while $21 \%$ of the couples in Burkina Faso, 21.4\% in Liberia, 21.9\% in Malawi and $19.2 \%$ in Zambia were in the middle wealth index.

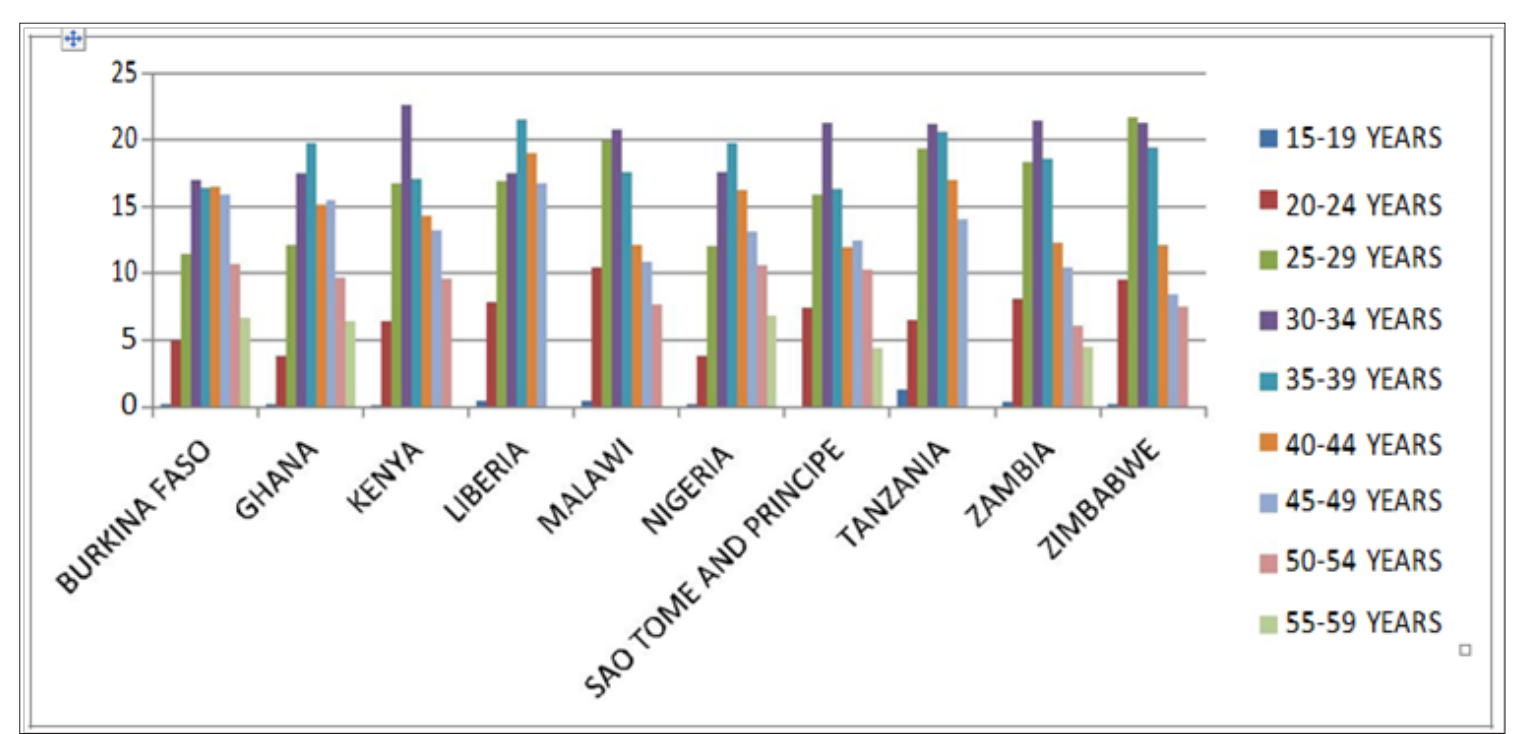

Figure 2b: Age Group of the Men.

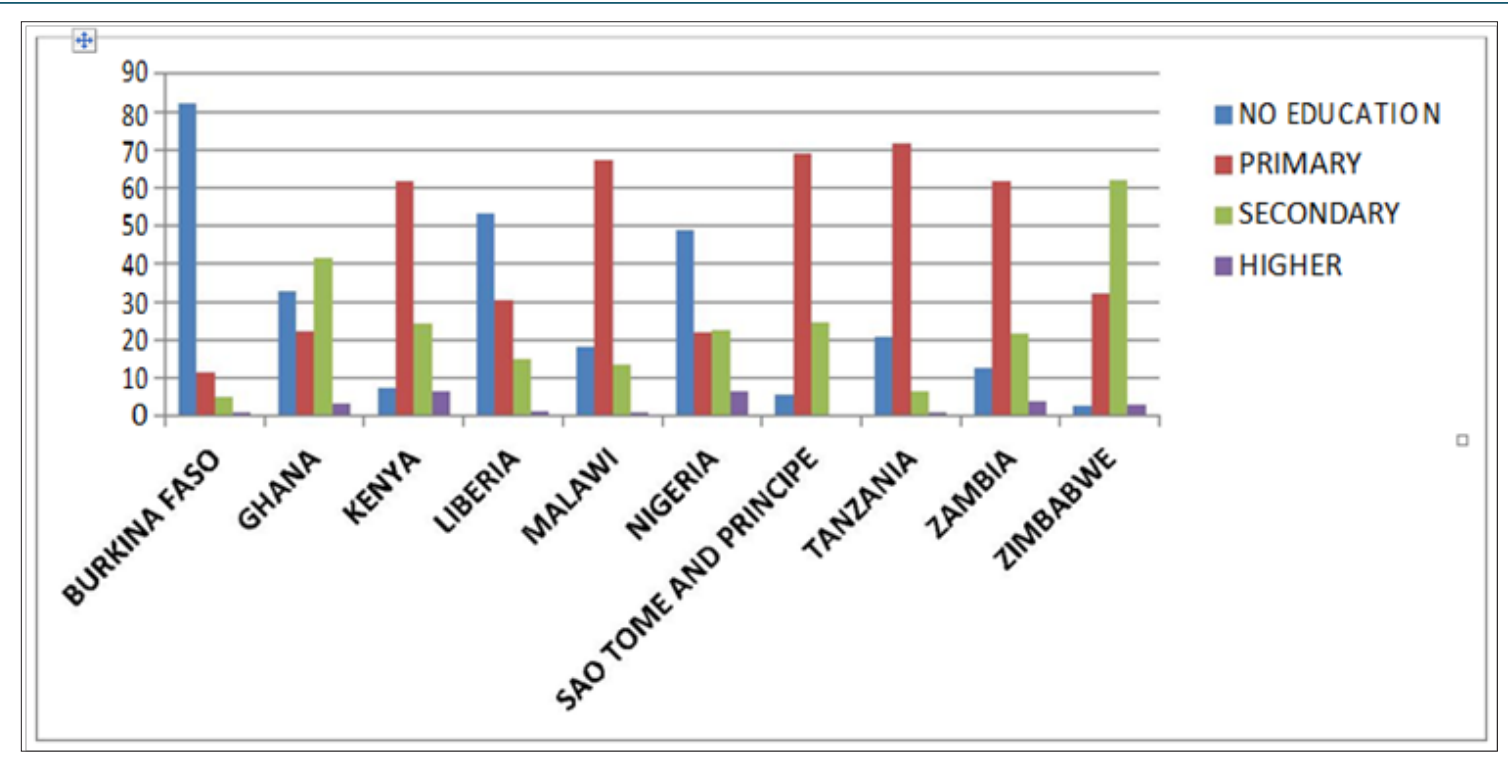

Figure 3A: Education Level of the Women.

\section{Prevalence of Intimate Partner Violence}

The prevalence of emotional IPV was lowest in Burkina Faso $(10.8 \%)$ and highest in Liberia (36.3\%). In Ghana, 31.4\% of the women reported experiencing emotional IPV while 32.3\% reported same in Burkina Faso. The percentage of emotional IPV is closest in Sao Tome and Principe and Zimbabwe with $25 \%$ of the women reporting emotional IPV in Sao Tome and Principe and 25.2\% in Zimbabwe. The percentage of women who reported sexual IPV ranged from as low as $1.2 \%$ in Burkina Faso to as high as $15.8 \%$ in Zimbabwe. Figures in Zambia and Malawi are closest to Zimbabwe with $15.6 \%$ of the women in Zambia and $15.4 \%$ of the women in the study population experiencing sexual violence from their husbands or partners. Zambian women reported the highest prevalence of physical IPV (44.9\%) while the lowest percentage of reported physical IPV was $11.5 \%$ in Burkina Faso. 


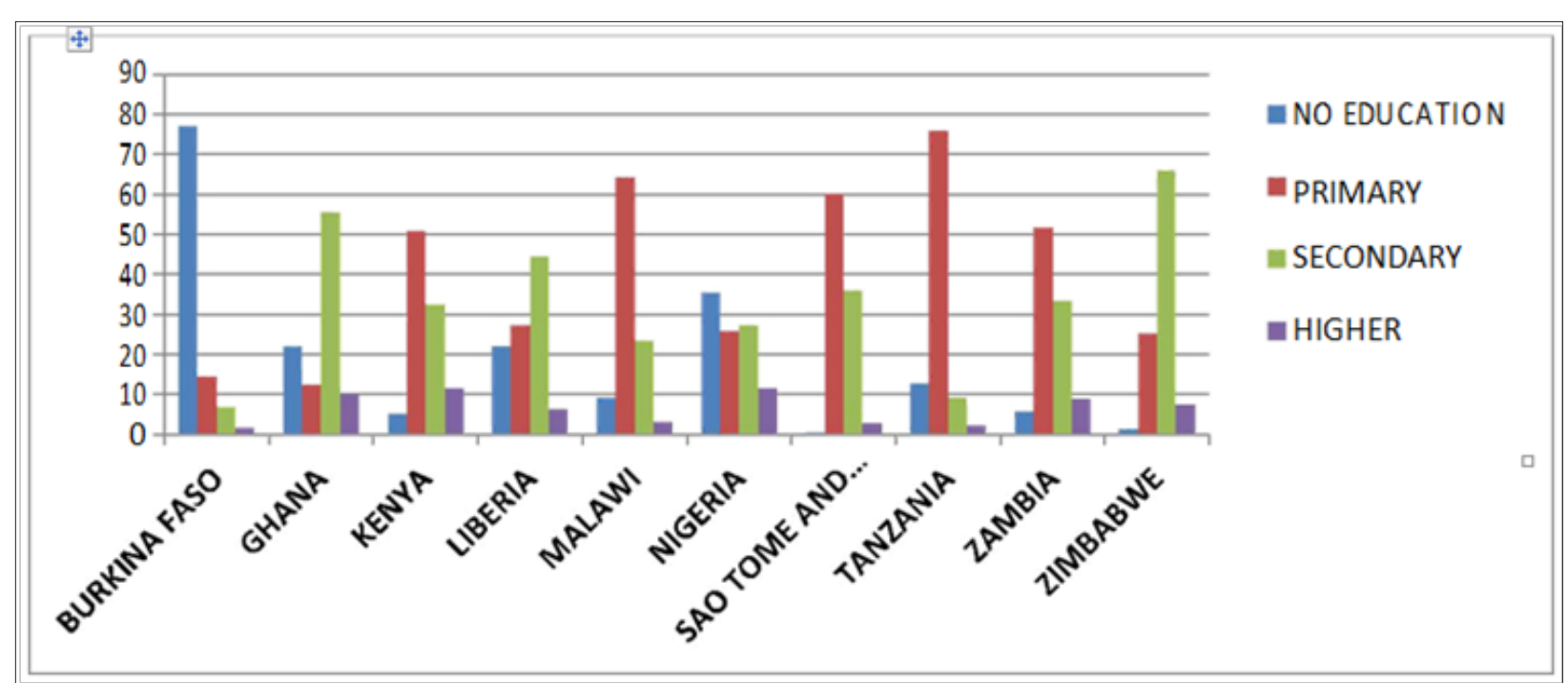

Figure 3b: Education Level of the Men.

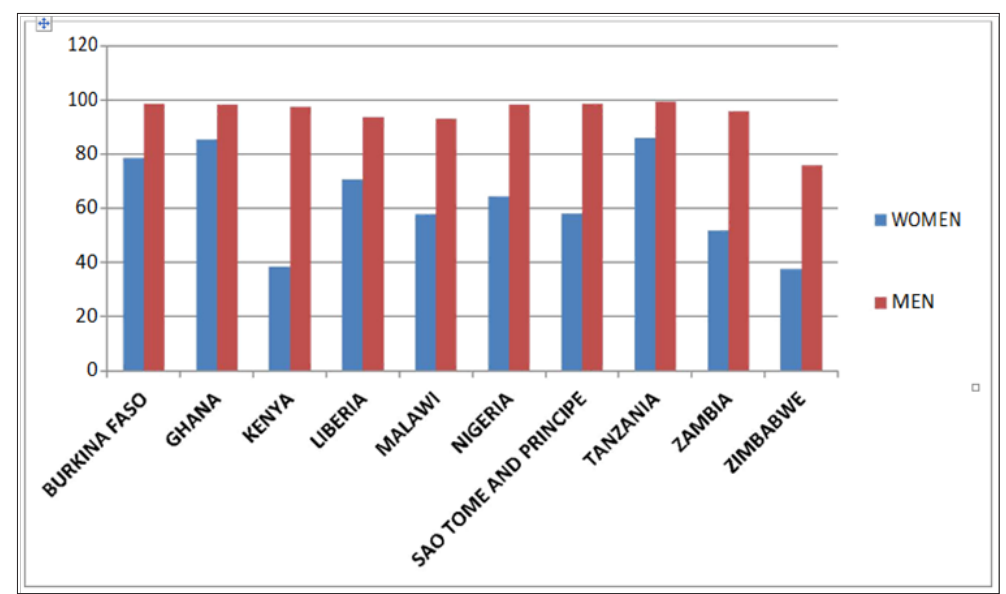

Figure 4: Employment Rate.

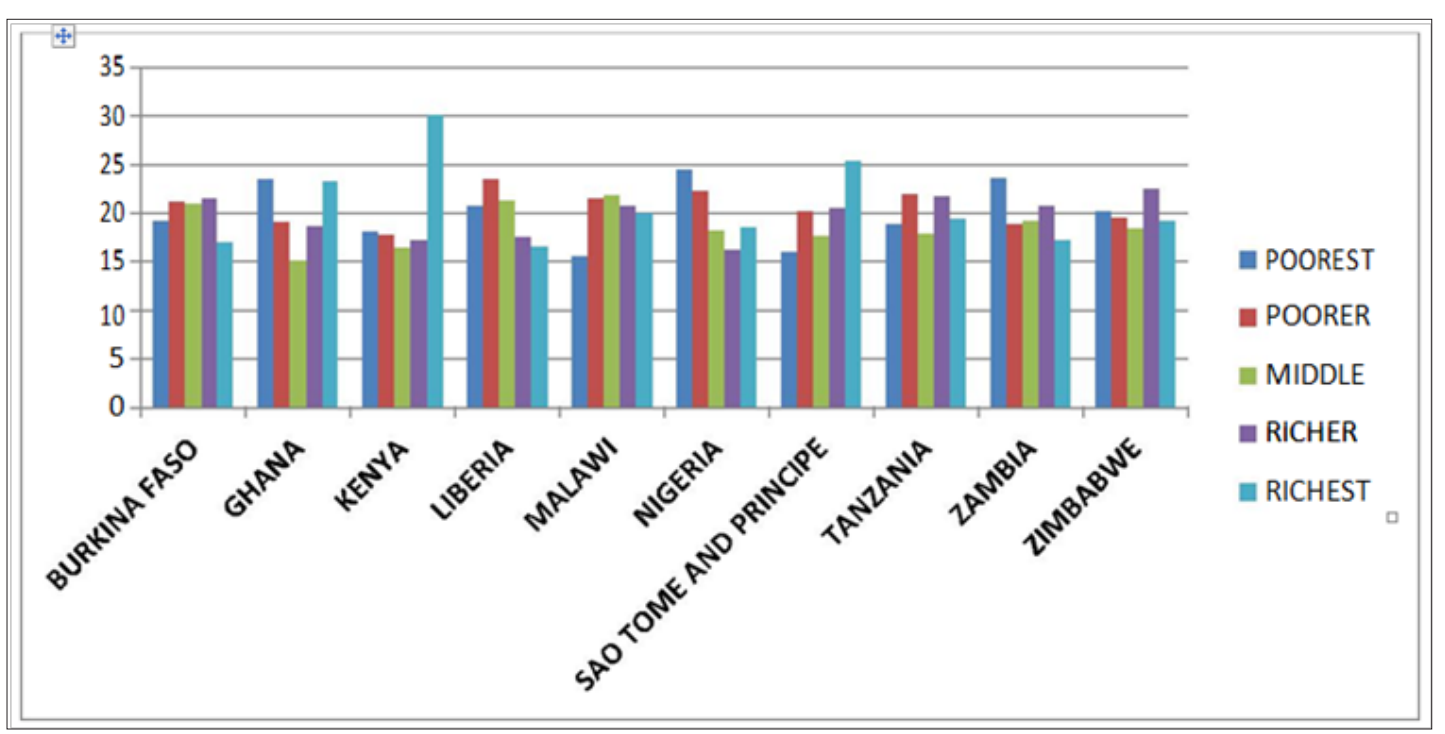

Figure 5: Wealth Index of Participants in the Study. 
In Kenya and Liberia, 32\% and 35\% of the women reported physical IPV. When all three forms of IPV were considered, prevalence rate was highest in Zambia (51.9\%) followed by Liberia (50.3\%) and lowest in Burkina Faso (16.7\%). In general, the preva- lence of physical and emotional IPV were higher than that of sexual IPV indicating that women are less likely to experience or report sexual IPV in sub-Saharan Africa (Figure 6).

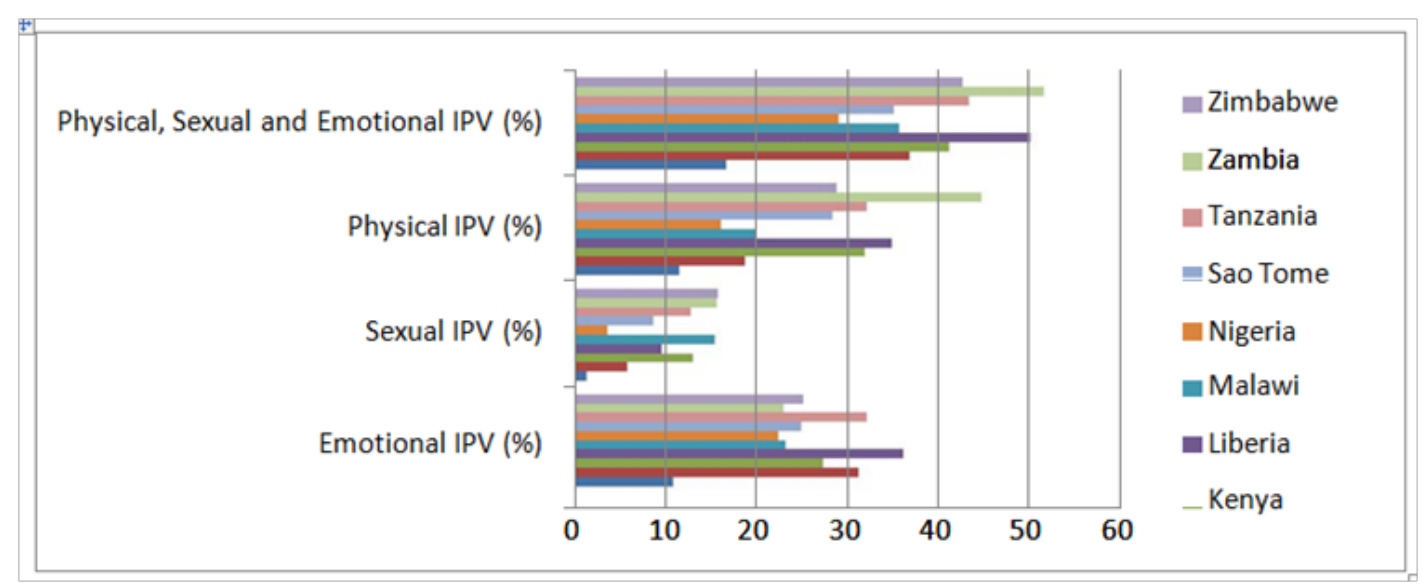

Figure 6: Prevalence of Intimate Partner Violence in sub -Saharan Africa.

\section{Prevalence of Alcohol-Related Intimate Partner Violence}

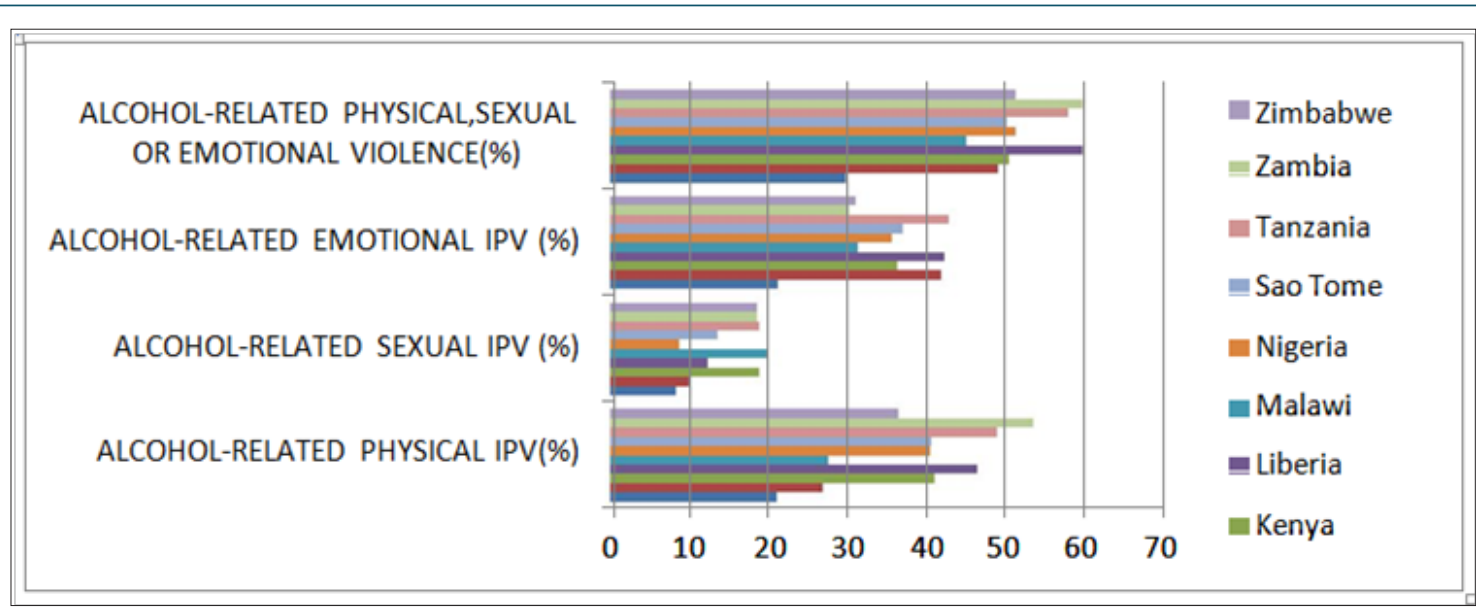

Figure 7: Prevalence of Intimate Partner Violence in sub -Saharan Africa.

Figure 7 shows the prevalence of alcohol-related intimate partner violence among married and cohabiting women in sub-Saharan Africa. The prevalence of alcohol related physical IPV was between 21.2 to $53.9 \%$ in this study. The highest prevalence rate of alcohol-related sexual violence was $19.9 \%$ in Malawi while the lowest prevalence of $8.8 \%$ was reported in Nigeria. The prevalence of alcohol-related emotional IPV ranged from $21.3 \%$ in Burkina Faso to $43.1 \%$ in Tanzania. When all three forms of IPV were considered, prevalence rates ranged from as $16.7 \%$ in Burkina Faso to as high as $51.9 \%$ in Zambia.

\section{Relationship between Alcohol and Intimate Partner Vi- olence}

The relationship between alcohol and intimate partner violence was investigated by conducting a logistic regression of intimate partner violence (physical, sexual or emotional IPV) and the four level drinking variables. The significance of the effect of each level of the drinking variable on IPV was assessed using the Wald's test. Odd ratios (Exp B) with the 95\% confidence intervals, standard error and Wald's test $\mathrm{p}$ values are presented. In Burkina Faso, the odds of women experiencing any form of IPV (physical, sexual or emotional IPV) is 1.7 times higher in women whose husbands never got drunk than in women whose husbands never drink while the odds of experiencing IPV is 2.23 (1.66-3.00) and 2.77 (1.82-4.21) greater in women whose husbands never got drunk compared to women whose husbands never drink in Nigeria and Sao Tome and Principe respectively. The Wald's test shows that there is a significant difference in the odds of experiencing IPV by women whose husbands drink but never got drunk and women whose husbands never drink. The results show that in Burkina Faso, Nigeria and Sao 
Tome and Principe, alcohol consumption rather than alcohol abuse is associated with intimate partner violence indicating that there is a linear relationship between alcohol and IPV in these countries.

In Ghana, the odd of perpetrating intimate partner violence is 2.29 (1.66-3.16) in men who got drunk sometimes and 3.36 (2.04$5.54)$ in men who got drunk often compared to non-drinkers. While the OR for experience of intimate partner violence is 7.12 (4.3011.81) in women whose husbands often abuse alcohol in Kenya, the OR for IPV by women whose husbands often get drunk is 2.72 (1.81-4.08) in Liberia. In Malawi, husbands/partners who get drunk sometimes are 1.45 times more likely to abuse their partners compared to men husbands or partners who do not drink alcohol while the odds of perpetrating IPV is 4 times higher in husbands who get drunk often. Wald's test $p$ values showed that there is no significant difference in the odds of women experiencing intimate partner violence in women whose husbands drink but never got drunk and women whose husbands never drink in Ghana, Kenya, Liberia, Malawi, Tanzania, Zambia and Zimbabwe. This indicates that it is alcohol abuse rather than drinking of alcohol that is associated with intimate partner violence. These full results are presented in Table 1 below.

Table 1: The effect of alcohol on intimate partner violence in sub-saharan Africa.

\begin{tabular}{|c|c|c|c|c|c|c|}
\hline & Burkina faso & & Nigeria & & Sao tome\&principe & B(se) \\
\hline & B(se) & Exp b(95\%ci) & B(se) & Exp b(95\%ci) & Exp b(95\%ci) \\
\hline No drinking(ref) & & & & & & \\
\hline Never drunk & $0.54(0.15)$ & $1.71(1.24-2.30)^{*}$ & $0.80(0.45)$ & $2.23(1.66-3.00)^{*}$ & $1.02(0.22)$ & $2.77(1.82-4.21)^{*}$ \\
\hline Sometimes drunk & $1.33(0.12)$ & $3.80(3.02-4.78)^{*}$ & $1.13(0.10)$ & $3.10(2.59-3.70)^{*}$ & $1.63(0.22)$ & $5.12(3.32-7.85)^{*}$ \\
\hline Often drunk & $1.78(0.19)$ & $5.91(4.04-8.65)^{*}$ & $1.99(0.20)$ & $7.31(4.91-10.88)^{*}$ & $2.60(0.36)$ & $13.4(6.58-27.27)^{*}$ \\
\hline
\end{tabular}

\begin{tabular}{|c|c|c|c|c|c|c|c|c|}
\hline $\begin{array}{c}\text { Threshold } \\
\text { effects }\end{array}$ & & & & & & & & \\
\hline & Ghana & & Kenya & & Liberia & & & \\
\hline & $\mathrm{B}(\mathrm{se})$ & Exp b(95\%ci) & $\mathrm{B}(\mathrm{se})$ & $\operatorname{Exp} b(95 \% c i)$ & $\mathrm{B}(\mathrm{se})$ & $\operatorname{Exp} b(95 \% c i)$ & & \\
\hline \multicolumn{9}{|l|}{$\begin{array}{l}\text { No drink- } \\
\text { ing(ref) }\end{array}$} \\
\hline Never drunk & $0.45(0.31)$ & $\begin{array}{c}1.58(0.85- \\
2.91)\end{array}$ & $0.25(0.84)$ & $\begin{array}{c}1.28(0.25- \\
6.68)\end{array}$ & & & & \\
\hline Sometimes & $0.83(0.16)$ & $\begin{array}{c}2.29(1.66- \\
3.16)^{*}\end{array}$ & $0.14(0.15)$ & $\begin{array}{c}1.14(0.86- \\
1.53)\end{array}$ & $0.56(0.10)$ & $\begin{array}{c}1.76(1.43- \\
2.15)^{*}\end{array}$ & & \\
\hline \multicolumn{9}{|l|}{ Drunk } \\
\hline \multirow[t]{3}{*}{ Often drunk } & $1.21(0.26)$ & $\begin{array}{c}3.36(2.04- \\
5.54)^{*}\end{array}$ & $1.96(0.26)$ & $\begin{array}{c}\text { 7.12(4.30- } \\
11.81)^{*}\end{array}$ & $1.00(0.21)$ & $\begin{array}{c}2.72(1.81- \\
4.08)^{*}\end{array}$ & & \\
\hline & Malawi & & Tanzania & & Zambia & & Zimbabwe & \\
\hline & $\mathrm{B}(\mathrm{se})$ & Exp b(95\%ci) & $\mathrm{B}(\mathrm{se})$ & Exp b(95\%ci) & $\mathrm{B}(\mathrm{se})$ & Exp b(95\%ci) & $\mathrm{B}(\mathrm{se})$ & $\operatorname{Exp} b(95 \%$ ci) \\
\hline \multicolumn{9}{|l|}{$\begin{array}{l}\text { No drink- } \\
\text { ing(ref) }\end{array}$} \\
\hline Never drunk & $0.15(0.26)$ & $\begin{array}{c}1.16(0.70- \\
1.94)\end{array}$ & $-0.11(0.43)$ & $\begin{array}{c}0.90(0.39- \\
2.08)\end{array}$ & $1.85(0.97)$ & $\begin{array}{c}0.16(0.02- \\
1.05)\end{array}$ & $-0.30(0.40)$ & $\begin{array}{c}0.74(0.34- \\
1.62)\end{array}$ \\
\hline $\begin{array}{l}\text { Sometimes } \\
\text { drunk }\end{array}$ & $0.37(0.09)$ & $\begin{array}{c}1.45(1.21- \\
1.73)^{*}\end{array}$ & $0.72(0.17)$ & $\begin{array}{c}2.05(1.46- \\
2.88)^{*}\end{array}$ & $0.43(0.09)$ & $\begin{array}{c}1.53(1.28- \\
1.84)^{*}\end{array}$ & $0.54(0.10)$ & $\begin{array}{c}1.72(1.43- \\
2.06)^{*}\end{array}$ \\
\hline Often drunk & $1.41(0.13)$ & $\begin{array}{c}4.10(3.17- \\
5.31)^{*}\end{array}$ & $1.64(0.25)$ & $\begin{array}{c}5.15(3.15- \\
8.40)^{*}\end{array}$ & $1.25(0.13)$ & $\begin{array}{c}3.50(2.72- \\
4.50)^{*}\end{array}$ & $1.25(0.15)$ & $\begin{array}{c}3.49(2.60- \\
4.79)^{*}\end{array}$ \\
\hline
\end{tabular}

The Strength of the Alcohol-Intimate Partner Violence Link in sub-Saharan

\section{a.Effect of Alcohol on Physical Intimate Partner Violence}

Figure 8 showed the result of the meta-analysis to determine the strength of the alcohol-physical intimate partner violence in ten sub-Saharan Africa countries. A total of 22,120 participants were used in the study. Of these numbers, 6,271 were in the group of women whose husbands drink abuse alcohol while 15,849 were in the group of were in the group of women whose husbands/partners do not abuse alcohol. While 2,576 women reported experienc- ing physical intimate partner violence in the first group, a total of 2,698 women reported physical IPV in the other group. The results show the test for heterogeneity, I2=93\% indicating a high degree of heterogeneity between the ten countries included in the analysis. The odds ratio is 2.91 (95\% CI (2.24-3.79) showing that there is a strong association between alcohol and physical intimate partner violence in sub-Saharan Africa. The overall effect $Z=7.96$ and was statistically significant $(\mathrm{p}<0.00001)$. This indicates that the strength of the alcohol-physical IPV link is strong in sub-Saharan Africa 


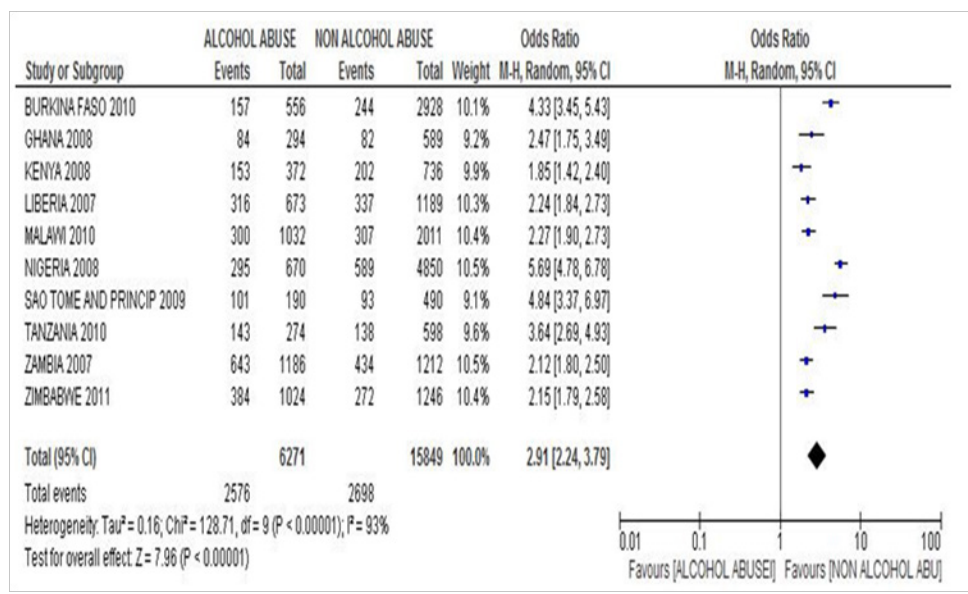

Figure 8: Effect of Alcohol on Physical IPV in SSA.

\section{b.Effect of Alcohol on Sexual Intimate Partner Violence}

The meta-analysis to determine the effect of alcohol on sexual IPV is presented in Figure 9. A total of 22,104 women were included in the study. 6,266 were in the alcohol abuse group while 15,838 were in the non- alcohol abuse group. Of the 6,266 women in the alcohol abuse group, 971 reported experiencing sexual IPV while 1,006 reported sexual IPV in the non-alcohol abuse group. The above results show an $\mathrm{I} 2$ value of $70 \%$ indicating heterogene- ity between studies. An OR of $2.15(95 \% \mathrm{CI}=1.78-2.60)$ means that women in sub-Saharan Africa are two times more likely to report sexual violence when their husbands/partners abuse alcohol than when they do not. An odd ratio of 2.15 shows that there is a small effect size for the alcohol-sexual violence link in sub-Saharan Africa. The overall effect $Z=7.87$ (0.00001) is highly significant indicating that the effect of alcohol on IPV is highly significant. These results imply that the strength of the alcohol-sexual IPV link is weak in sub-Saharan Africa.

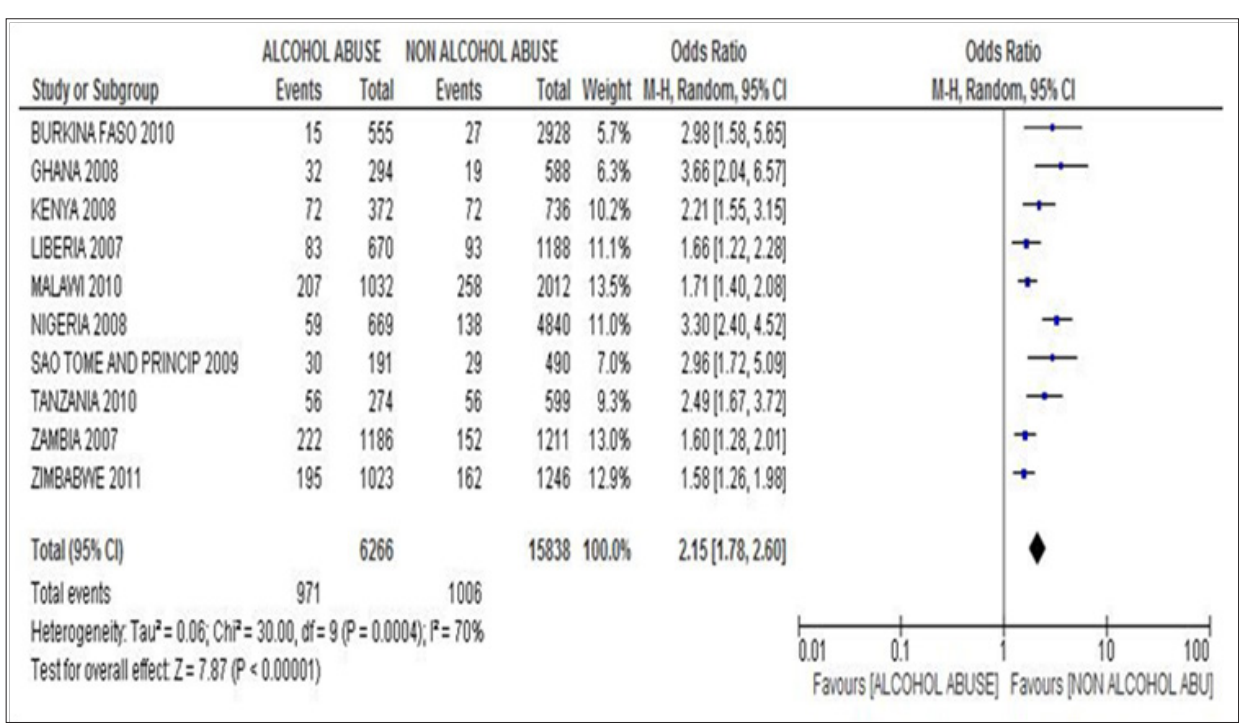

Figure 9: Effect of Alcohol on Sexual Intimate Partner Violence in sub-Saharan Africa.

\section{c.Effect of Alcohol on Emotional Intimate Partner Violence}

A total of 22,112 respondents took part in the study to determine the effect of alcohol on emotional intimate partner violence. A total of 5,151 participants reported experiencing emotional intimate partner violence. Of this number, 2,182 reported that their husbands abuse alcohol while 2,969 reported that their husbands never abuse alcohol. The result of the meta-analysis is presented in Figure 10 below. The results above show that women whose husbands abuse alcohol are 2.36 times more likely to report emotional intimate partner violence in sub-Saharan Africa $\mathrm{OR}=2.36$
(95\%1.96-2.83). This means that there is a moderate effect size for the association between alcohol and emotional intimate partner violence in sub-Saharan Africa. An I2 value of $85 \%$ indicates high heterogeneity between studies. The overall effect $\mathrm{Z}$ was highly significant $(Z=9.15, \mathrm{p}<0.00001)$

\section{Discussion}

\section{Prevalence of Intimate Partner Violence in SSA}

In this study, the prevalence of physical intimate partner violence is $44.9 \%$ in Zambia, $28.9 \%$ in Zimbabwe and $19.9 \%$ in Mala- 
wi. These values are similar to the $45 \%, 28 \%$ and $20 \%$ reported by Hindin et al. [15] for Zambia, Zimbabwe and Malawi respectively. On the other hand, the $32 \%$ and $32.2 \%$ prevalence rates reported for Kenya and Tanzania herein are lower than the 39\% reported by Hindin et al. [15] and the $48 \%$ observed by Tumwesigye et al. [16] for Uganda which is a neighboring East African country. The percentage of physical IPV in other countries in this study ranged from between $11.5 \%$ in Burkina Faso to $28.5 \%$ in Sao Tome and Principe.
While some of these rates are similar to figures reported in other sub-Saharan African countries, others are less. For instance, the $28.5 \%$ prevalence reported here is similar to the $29 \%$ reported for Rwanda [15]. However, the $11.5 \%$ reported for Burkina Faso is lower than rates reported anywhere in sub-Saharan Africa but similar to the $12 \%$ reported for Haiti [3]. These differences in prevalence rates may be as a result of the questions used to assess physical IPV.

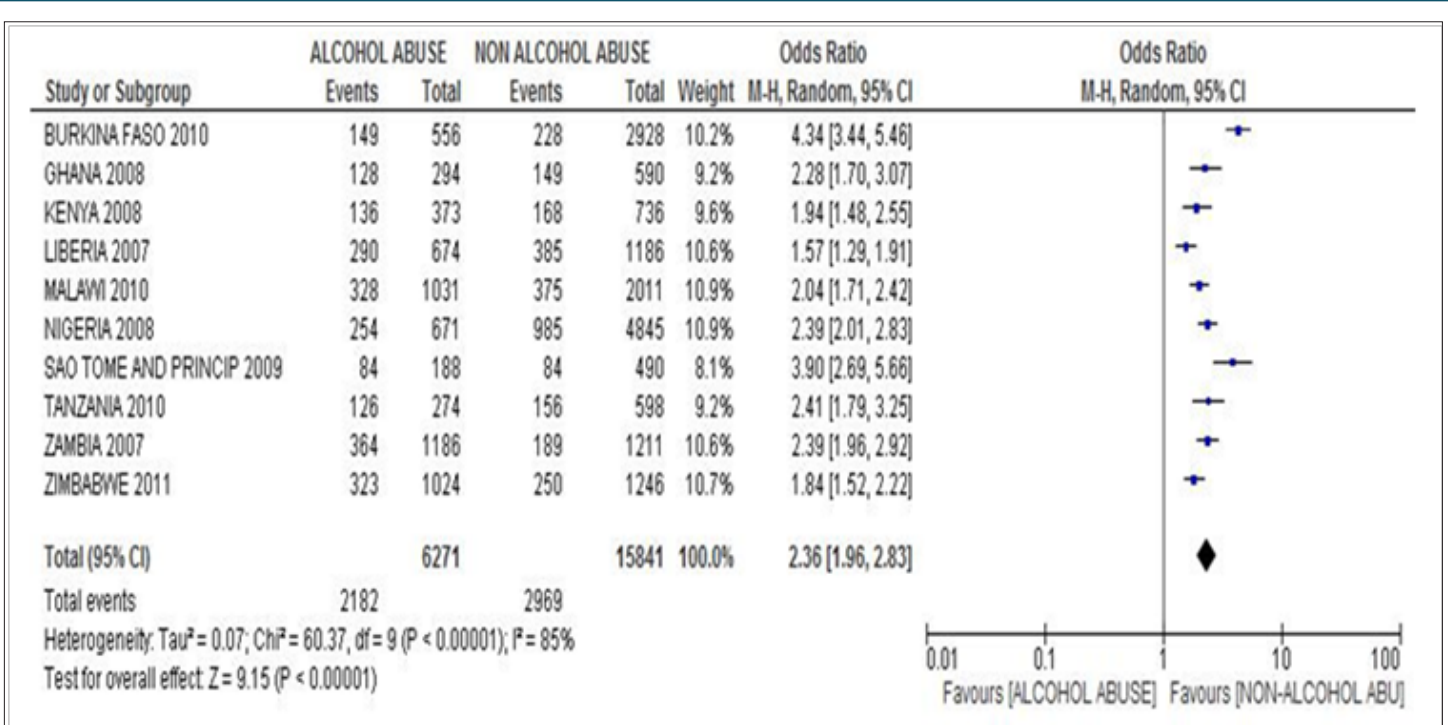

Figure 10: Effect of Alcohol on Emotional Intimate Partner Violence in SSA.

This study asked respondents whether husband/partner has ever threatened or attacked them with a gun/knife or other weapons while the Hindin et al. [15] separated this question into threat and actual attack. Combining these two questions would mean that women who have experienced both threat and the act can only give one response to both questions leading to the lower rates obtained in this study. In this study, $16.1 \%$ of women in Nigeria reported physical IPV. This is slightly higher than the $15 \%$ reported by Antai et al. [17] using the same study data. While the Antai et al. [17] study used the individual recode which is a sample of legally married women, the present study used a sample of currently married/cohabiting women. This is consistent with findings that the prevalence of IPV is higher in currently married than legally married women [15]. In spite of these observed differences in the prevalence of physical IPV in some sub-Saharan African countries in this study, the observed prevalence of 11.5 to $44.9 \%$ in this study is consistent with that reported in other studies [2,3,16-18].

Apart from physical IPV, the prevalence of sexual and emotional IPV was also examined. Results indicated that the rates of sexual IPV ranged from 1.2\% in Burkina Faso to $15.8 \%$ in Zimbabwe. These rates are similar to the 3 to $16 \%$ prevalence rates reported in existing literatures for sub-Saharan Africa $[15,17]$. The prevalence of emotional IPV in this study ranged from $10.8 \%$ in Burkina Faso to $36.3 \%$ in Liberia. This is higher than the prevalence rates of 10.4 to $22.7 \%$ reported elsewhere [19]. When all three forms of IPV were considered, the prevalence rates increased for all ten coun- tries and were higher than that reported in previous studies. For example, Hindin et al. [15] reported a prevalence of $45 \%$ for Zambia in their studies while this study shows a prevalence of $51.9 \%$ for Zambia when all forms of IPV were considered. It can thus be argued that studies investigating only one form of IPV result in an under estimation of the magnitude of IPV. Overall, the prevalence of sexual violence in this study was consistently lower than that reported for physical and emotional violence across all countries included in this work.

\section{Prevalence of Alcohol-Related Intimate Partner Violence}

The prevalence of alcohol IPV was between $29.9 \%$ to $60.1 \%$ when of physical, sexual or emotional IPV is considered. These rates are higher than the 33.9 to $49.5 \%$ reported for countries in sub-Saharan Africa [15,16] and 10.5 to $55 \%$ reported elsewhere [2]. However, this is lower than the $65 \%$ rate reported in South Africa [2]. The higher rates reported in this study is as a result of the fact that this study investigated the experience of all three forms of IPV indicating that previous studies may have been subject to an under reporting of prevalence rates of IPV as only physical or sexual IPV are usually investigated. The lower prevalence of alcohol-related IPV in this study compared to the $65 \%$ prevalence reported in South Africa can be explained by the fact that while the present study investigated the prevalence of women ever experiencing alcohol-related IPV, the South African study reported prevalence for the past twelve months. 
Following the same trend as IPV, it is possible that the occurrence of current alcohol-related violence may be higher than past occurrence. Women who have experienced alcohol-related violence may recall these incidents more since they are more recent than if they took place a long time ago. Another possible explanation for this is that in South Africa, it is believed that alcohol causes aggression and this has led men to drink in order to carry out violent acts [2] thereby increasing the rate of alcohol-related violence in South Africa compared to other sub-Saharan African countries.

\section{The Relationship between Alcohol and Intimate Partner Violence in SSA}

In all ten countries, alcohol was consistently linked to women's experience of intimate partner violence with the nature of the relationship varying across different countries. The findings of this study showed that in seven out of the eleven countries (Ghana, Kenya, Liberia, Malawi, Tanzania, Zambia and Zimbabwe), there was no significant difference in the odds of experiencing IPV in women whose husbands/partners never consumed alcohol and those whose husbands/partners never got drunk. This is consistent with the findings in several literatures $[2,3,16]$. This can be explained by the fact that for alcohol to significantly increase the odds of perpetrating intimate partner violence, alcohol consumption has to surpass a particular amount or rate of consumption.

This explanation is consistent with that put forward by proponents of the threshold effect who argue that it is not alcohol consumption per se that contributes to intimate partner violence but alcohol abuse. Conversely, in Burkina Faso, Nigeria and Sao Tome and Principe, women whose husbands consumed alcohol but never got drunk were significantly more likely to experience any of physical, sexual or emotional intimate partner violence than women whose husbands never consumed alcohol. This is similar to the report of Bangdiwala et al. [20] who reported a strong linear relationship for the alcohol-IPV link. These results are in agreement with the justification put forward by the proponents of the linear effect conceptualization who maintain that alcohol abuse increases the odds of intimate partner violence and that these odds increases with increase in the quantity of alcohol consumed.

In Kenya alone, the odds of alcohol increasing the odds of intimate partner violence only reached statistical significance in the women whose husbands/partners often got drunk. This indicates that in Kenya, it is the frequency of alcohol abuse and not abuse alone that contributes to the alcohol-IPV link. Drawing from the multiple threshold conceptualisation [21], it is possible that the likelihood of experiencing intimate partner violence in Kenya is already very high that alcohol consumption or less frequent alcohol abuse does not significantly contribute to an increased odd of intimate partner violence while more frequent alcohol abuse may increase the frequency and severity of intimate partner violence in this sample thereby increasing the likelihood of intimate partner violence in this group.
The findings of this study support both the linear and threshold conceptualisation of the alcohol-IPV link. This suggests that the type of relationship between alcohol and intimate partner violence varies across countries in sub-Saharan Africa with most countries showing a threshold effect. This observed difference could be as a result of differences in drinking pattern. In the seven countries where threshold effects were observed, large percentages (86.3 to $99.1 \%$ ) of men who drink get drunken showing drinking cultures that are supportive of alcohol abuse.

\section{Strength of Alcohol-Intimate Partner Violence Link in SSA}

A meta-analysis of the ten countries included in this study showed an odds ratio of 2.91 (95\%CI, 2.24-3.39) for physical IPV which shows that women whose husbands abuse alcohol are almost thrice as likely to experience IPV than women whose husbands do not abuse alcohol. This shows a strong association between alcohol and intimate partner violence in sub-Saharan Africa. This is consistent with the strong association reported in a meta-analysis by Gil-Gonzalez et al. [22]. The strong association between alcohol and physical IPV in this study can be explained by the culture of masculinity in sub-Saharan Africa. It is considered masculine for men to drink and it is also seen as a thing of pride for a man to be feared and respected by his wife. It is also generally acceptable for men to physically reprimand their wives if they feel that the women have erred in anyway.

On the other hand, a small but significant relationship was observed for alcohol and sexual intimate partner violence in this study. This is similar to the report of Tang and Lai [23] who reported a significant but weak association between alcohol abuse and sexual intimate partner violence. This weak association could be as a result of the fact that in African society, sex is considered a private matter not to be discussed with strangers. There is also shame associated with being raped and made to perform unwanted sexual acts and women are often blamed for it and this may result in under reporting. Women may also consider that being made to perform any form of sexual act is acceptable so long as it is within a marriage or committed relationship and may not consider this as IPV. For example, it is commonly believed in Africa that a man cannot rape his wife. Hence forced sexual intercourse in a marriage is not considered rape but seen as a normal part of the relationship. When the severity of intimate partner. Based on the results from this research, it can be argued that the strength of the alcohol-IPV link in sub-Saharan Africa depends on the type of IPV measured.

\section{Conclusion}

The purpose of this research work is to identify the relationship, strength of relationship between alcohol and intimate partner violence in sub-Saharan Africa. Results of this study show that alcohol is consistently associated with intimate partner violence in all ten countries included in this study with the strength of this 
relationship depending on the form of intimate partner violence. While a linear relationship was found between alcohol and intimate partner violence in Burkina Faso, Nigeria and Sao Tome and Principe, a threshold effect was observed in the remaining seven countries with the odds of alcohol-related intimate partner violence increasing with increase in alcohol consumption and alcohol abuse. The strength of the alcohol-intimate partner violence link was also found to be dependent on the effects of other variables in some countries with the direction of moderation different in the countries where these moderation effects are present.

The results also show that when the three forms of IPV were measured, the prevalence of both IPV and alcohol-related IPV were higher than that of existing literatures suggesting that studies of IPV estimating only one or two forms of IPV are subject to under estimation of the magnitude of his huge public health problem. These findings have potentially important implications for public health promotion, policy and practice. The implication of the findings of this research is that interventions for tackling alcohol-related IPV should be multifaceted and should address behavioural, cultural and social change

\section{Study Limitations}

Despite the above strengths, the result of this study should be interpreted bearing in mind several limitations. First, because the information on problem drinking was provided by women, there is the possibility of misclassification bias. Responses were not taken from men about their drinking and IPV perpetration and this would have been valuable in corroborating the women report. There is also a tendency for recall bias as IPV may have taken place at a different time from alcohol abuse. Classification of drinking as sometimes or often drunk makes the report subjective rather than objective and may have led to results showing a stronger relationship between alcohol and IPV in sub-Saharan Africa. This is because studies have shown that studies assessing alcohol abuse is more associated with IPV than those measuring quantities [24].

Even though the results of this research are to a great extent consistent with those reported for community samples in other studies, it cannot be generalised to clinical samples of alcohol abusers or IPV perpetrators because studies have shown that the magnitude of the association between alcohol and IPV is stronger in clinical than community samples [24]. Finally, because this study analysed data from cross sectional studies which are ecological in nature, it is difficult to know if problem drinking took place before IPV or vice versa. It is possible that perpetrators of IPV are more likely to misuse alcohol or that individual's use alcohol to cope with stressful life events hence causality cannot be assumed.

\section{References}

1. World Health Organization (2012) Intimate Partner Violence and Alcohol Fact sheet.

2. World Health Organization (2010) Preventing intimate partner and sex- ual violence against women: Taking action and generating evidence. Injury Prevention 16: 359-360.

3. Gage AJ (2005) Women's experience of intimate partner violence in Haiti. Social science \& medicine 61(2): 343-364.

4. Krug EG, Mercy JA, Dahlberg LL, Zwi AB (2002) The world report on violence and health. The lancet 360 (9339): 1083-1088.

5. Stith SM, Smith DB, Penn CE, Ward DB, Tritt D, et al. (2004) Intimate partner physical abuse perpetration and victimization risk factors: A meta-analytic review. Aggression and Violent Behavior 10(1): 65-98.

6. Uthman OA, Lawoko S, Moradi T (2010) Sex disparities in attitudes towards intimate partner violence against women in sub-Saharan Africa: a socio-ecological analysis. BMC Public Health 10(1): 223-223.

7. Foran HM, Heyman RE, Slep AMS, Snarr JD (2012) Hazardous alcohol use and intimate partner violence in the military: Understanding protective factors. Psychology of Addictive Behaviours 26(3): 471-483.

8. Abrahams N, Jewkes R, Laubscher R, Hoffman M (2006) Intimate partner violence: prevalence and risk factors for men in Cape Town, South Africa. Violence and victims 21(2): 247-264.

9. Kimuna SR, Djamba YK (2008) Gender Based Violence: Correlates of Physical and Sexual Wife Abuse in Kenya. Journal of Family Violence 23(5): 333-342.

10. Welman C, Huysamen GK, Kruger F, Mitchell B (2005) Research methodology. Oxford: Oxford University Press, UA.

11. Littell JH, Corcoran J, Pillai VK (2008) Systematic reviews and meta-analysis. Oxford University Press. New York, USA.

12. Borenstein M, Hedges LV, Higgins J, Rothstein HR (2009) Power Analysis for Meta-Analysis. Introduction to Meta-analysis pp. 257-276.

13. Field A (2005) Discovering statistics using SPSS: and sex, drugs and rock'n'roll .SAGE Publications, London.

14.Demographic and Health Survey (2012) Questionnaires. http:// www.measuredhs.compubs/pdf/DHSQ6/DHS6_Questionnaires_5Nov2012DHSQ6.pdf

15. Hindin MJ, Kishor S, Ansara DL (2008) Intimate partner violence among couples in 10 DHS countries: Predictors and health outcomes. Macro International Incorporated.

16. Tumwesigye NM, Kyomuhendo GB, Greenfield TK, OWanyenze RK (2012) Problem drinking and physical intimate partner violence against women: evidence from a national survey in Uganda. BMC public health 12(1): 399-399.

17. Antai D (2011) Controlling behaviour, power relations within intimate relationships and intimate partner physical and sexual violence against women in Nigeria. BMC public health 11(1): 511-511.

18. Kishor S, Johnson K (2004) Risk factors for the experience of domestic violence. Profiling domestic violence: a multi-country study. Calverton, Maryland: Measure DHS ORC Macro pp. 27-52.

19. Bazargan Hejazi S, Medeiros S, Mohammadi R, Lin J, Dalal K, et al. (2013) Patterns of intimate partner violence: a study of female victims in Malawi. Journal of injury \& violence research 5(1): 38-50.

20. Bangdiwala SI, Taylor BB, Shankar V (2010) Alcohol drinking behaviour patterns and intimate partner violence. Injury Prevention 16(Supp 1): A187-A187.

21. Fals Stewart W, Leonard K, Birchler G (2005) The occurrence of male-tofemale intimate partner violence on days of men's drinking: The moderating effects of antisocial personality disorder. Journal of consulting and clinical psychology 73(2): 239-248. 
22. Gil Gonzalez D, Vives Cases C, Alvarez Dardet C, Latour Perez J (2006) Alcohol and intimate partner violence: do we have enough information to act? The European Journal of Public Health 16(3): 278-284.

23. Tang CS, Lai BP (2008) A review of empirical literature on the prevalence and risk markers of male-on-female intimate partner violence in contemporary China, 1987-2006. Aggression and Violent Behavior[online] 13(1): 10-28.

24. Foran HM, O Leary KD (2008) Alcohol and intimate partner violence: A meta-analytic review. Clinical psychology review 28(7): 1222-1234.

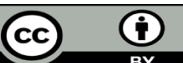

This work is licensed under Creative

Commons Attribution 4.0 License

To Submit Your Article Click Here : Submit Article

DOI: $10.32474 /$ RRHOAJ.2018.01.000110

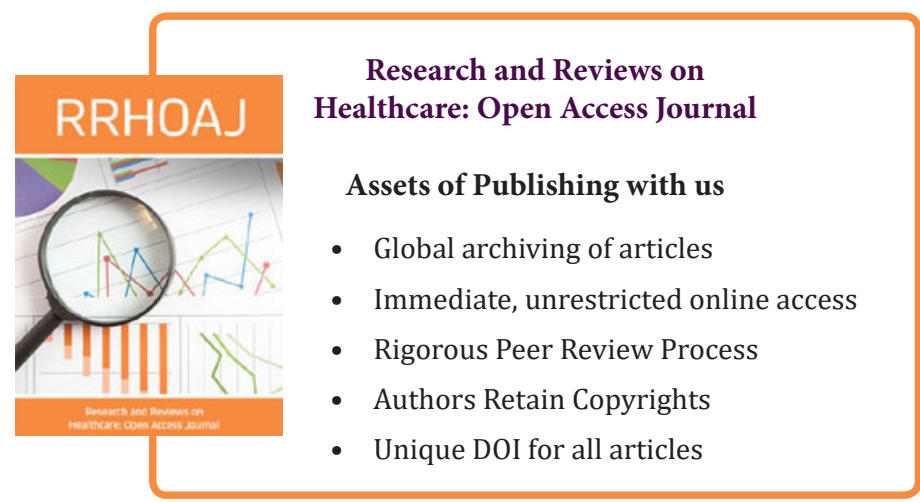

